\title{
Effective Distances of Exothermic Charge-Transfer Reactions in the Excited State ${ }^{\dagger}$
}

\author{
Sïlvia M. B. Costa,* A. L. Maçanita, \\ Centro de Quimica Estrutural, Complexo I, Instituto Superior Tëcnico, Av. Rovisco Pais, 1096 Lisboa Codex, \\ Portugal
}

\author{
and S. J. Formosinho
}

Departamento de Quimica, Universidade de Coimbra, 3000 Coimbra, Portugal (Received: August 16, 1983; In Final Form: January 10, 1984)

\begin{abstract}
A study of intermolecular, exothermic charge-transfer interactions is described. A distance dependence on the unimolecular charge-transfer rate constant $k_{\mathrm{ct}}(R)$ is observed in viscous solvents where transfer at longer distances than the collisional one can compete with dissociation of an encounter complex. A comparative study of the quenching phenomena, occurring in a family of redox partners of aromatic esters and amines, was carried out in nonviscous solvents (hexane and acetonitrile) and viscous solvents (liquid paraffin/heptane mixtures and nonionic micelles of Triton X-100). In the latter, the experimental reactional distances obtained are discussed in terms of effective distances $R_{\text {eff }}$, which are related to an average value of $k_{\mathrm{ct}}\left(R_{\text {eff }}\right)$ for the reactant pair distribution function. A correlation of these distances with either the free activation energy, $\Delta G^{*}$, or the diffusion coefficient $D$ enables the estimation of an order of magnitude of parameters such as the average radii of donor and acceptor orbitals, $L=(2.7 \pm 0.2) \times 10^{-8} \mathrm{~cm}$, a preexponential factor at the collisional distance, $k^{\circ}\left(R_{\mathrm{c}}\right)=3.7 \times 10^{11}$ $\mathrm{s}^{-1}$, an intrinsic reorganization parameter $\lambda=0.64 \mathrm{eV}$, and an electronic matrix element $V\left(R_{\mathrm{c}}\right) \simeq 10^{-3} \mathrm{eV}$ for the nonpolar media studied. An equation for $k_{\mathrm{ct}}(R), k_{\mathrm{ct}}(R)=k^{\circ}{ }_{\mathrm{ct}}\left(R_{\mathrm{c}}\right) \exp \left[-2\left(R-R_{\mathrm{c}}\right) / L\right] \exp \left(-\Delta G^{*} / k_{\mathrm{B}} T\right)$, derived within the framework of a semiclassical theory, is used to discuss these interactions as equally nonadiabatic reactions to which an extended Marcus formalism is applied.
\end{abstract}

\section{Introduction}

Electronically excited states very often interact with a variety of quenchers via an electron-transfer mechanism. In nonpolar solvents, many systems exhibit an exciplex emission which has made possible rather detailed photophysical studies ${ }^{1,2}$ and provided a mechanism for the quenching of excited singlet ${ }^{1} \mathrm{~A}$ * by some quencher $\mathrm{Q}$. Indeed, the formation of excited-state complexes is a well-known phenomenon, and its mechanism and kinetic and thermodynamic aspects have been extensively documented. ${ }^{3-7}$ In other systems, fluorescence quenching is not accompanied by the observation of an exciplex emission, but radical ions are found in polar solvents. ${ }^{8}$

Kinetic data available from electron-transfer studies deal mainly with correlations of rate constants with the standard Gibbs free energy change of the electron-transfer $\operatorname{step}^{7,9,10}$ from which it is possible to evaluate the standard free energy of activation $\Delta G^{\neq 11}$ and discuss the so-called intrinsic reorganizational parameter $\lambda$ in the context of Marcus theory. These studies are particularly relevant for nondiffusion-controlled reactions for which the slow electron-transfer process is the rate-determining step.

Another physical property of the medium, the viscosity, can also markedly affect rate constants of reactions which take place near the diffusion-controlled limit $\left(k_{\mathrm{q}} \simeq k_{\text {diff }}\right) .{ }^{12}$ In such cases reactional distances $R^{\prime}$ are often larger than the collisional ones, $R_{\mathrm{c}}$, and values of $R^{\prime}$ and diffusion coefficients $\left(D_{\mathrm{AQ}}\right)$ obtained with the classical Smoluchowski model ${ }^{13}$ are inconsistent with the experimental data. The differences have been accounted for by an exchange interaction 12 or by an additional "pseudo-static" quenching arising from quencher molecules located at distances between $R_{\mathrm{c}}$ and $R^{\prime}$ from ${ }^{1} \mathrm{~A}^{*},{ }^{14}$ still in the framework of the Smoluchowskii model.

Ware and co-workers ${ }^{15}$ have extended their analysis to radiation boundary conditions, but they do not correlate $R^{\prime}$ or the intrinsic electron-transfer rate constant with relevant parameters such as redox potentials, dielectric constants, and viscosities. Pilling and Rice ${ }^{16}$ incorporated a distance-dependent sink term in the diffusion equation and solved it in the steady state. They found out that for a low viscosity range $\left(D \simeq 10^{-5} \mathrm{~cm}^{2} \mathrm{~s}^{-1}\right)$ the effective encounter distance $R_{\text {eff }}$ is equal to the collisional distance $R_{\mathrm{c}}$, but for higher

${ }^{+}$Presented in part at the 8th IUPAC Symposium on Photochemistry, Seefeld, Austria, 1980 (Abst. Proc., p 106). viscosities the diffusion is too slow, so that energy (or electron) transfer can compete with diffusion and occur at distances larger than $R_{\mathrm{c}}$. This model was applied to studies of phenanthrene triplet quenching by $\mathrm{Cu}^{2+}$ and $\mathrm{Eu}^{3+}$ via an energy-transfer mechanism, and indeed $R_{\text {eff }}$ was found to increase with viscosity. ${ }^{17}$

The question as to how does the electron-transfer rate constant depend on the distance has been theoretically addressed by several authors. ${ }^{18}$ One experimental approach has been the use of rigid matrices, ${ }^{19}$ and exothermic rate restrictions have been demonstrated in such studies. ${ }^{20}$

On the other hand, many studies have found diffusion-limited rate constants which extend to quite negative $\Delta G^{\circ}{ }_{s}{ }^{7 b, 21,22}$ Re-

(1) W. R. Ware and H. P. Richter, J. Chem. Phys., 48, 1595 (1968),

(2) (a) T. Okade, H. Matsui, H. Oohari, M. Matsumota, and N. Mataga, J. Chem. Phys., 49, 4717 (1968); (b) K. Yoshihara, T. Kasya, A. Inoue, and S. Nagakura, Chem. Phys. Lett., 9, 469 (1971).

(3) H. Leonhardt and A. Weller, Ber. Bunsenges. Phys. Chem., 67, 791 (1963)

(4) H. Knibbe, D. Rehm, and A. Weller, Ber. Bunsenges. Phys. Chem., 73, 839 (1969).

(5) D. Rehm and A. Weller, Z. Phys. Chem. (Wiesbaden), 69, 83 (1970).

(6) T. R. Evans, J. Am. Chem. Soc., 93, 2081 (1971).

(7) (a) D. Rehm and A. Weller, Ber. Bunsenges. Phys. Chem., 73, 834

(1969); (b) D. Rehm and A. Weller, Isr. J. Chem., 8, 259 (1970).

(8) S. M. B. Costa and E. C. C. Melo, J. Chem. Soc., Faraday Trans. 2, 76, 1 (1980)

(9) V. Balzani and F. Scandola in "Photochemical Conversion and Storage of Solar Energy" J. S. Connolly, Ed., Academic Press, New York, 1981. (10) F. Scandola, V. Balzani, and G. B. Schuster, J. Am. Chem. Soc., 103, 2519 (1981)

(11) R. A. Marcus, J. Chem. Phys., 43, 679 (1965), and references cited therein.

(12) J. B. Birks and M. S. S. C. P. Leite, J. Phys, B, 3, 417 (1970),

(13) (a) M. V. Smoluchowski, $Z$. Phys. Chem., Stoechiom. Verwandts-

chaftsl., 92, 129 (1917); (b) R. M. Noyes, Prog. React. Kinet, 1, 31 (1961).

(14) J. C. Andre, M. Niclause, and W. R. Ware, Chem. Phys., 28, 371 (1978).

(15) (a) J. C. Andre, M. Bouchy, and W. R. Ware, Chem. Phys., 37, 103

(1979); (b) J. C. Andre, M. Bouchy, and W. R. Ware, ibid., 37, 117 (1979).

(16) (a) M. J. Pilling and S. A. Rice, J. Chem. Soc., Faraday Trans. 2, 71, 1563 (1975); (b) ibid., 72, 792 (1976).

(17) (a) E. J. Marshall and M. J. Pilling, J. Chem. Soc., Faraday Trans.

2, 579 (1978); (b) P. R. Butler and M. J. Pilling. Chem. Phys, 39, 33 (1979).

(18) J. Jortner, J. Am. Chem. Soc, 102, 6676 (1980), and references cited therein.

(19) J. R. Miller and J. V. Beitz, J. Chem. Phys., 74, 6746 (1981).

(20) J. V. Beitz and J. R. Miller, J. Chem. Phys., 71, 4579 (1979). 
cently, Marcus has discussed high exothermic electron-transfer reactions occurring over a range of distances. ${ }^{23}$

We have previously studied the photokinetics of several donor/acceptor systems in both nonviscous solvents ${ }^{24}$ as well as in nonionic Triton-X micelles. ${ }^{25}$ In the latter systems we found that a decrease of the standard free energy change $\Delta G^{\circ}$ is accompanied by an increase of reactional distances obtained from experimental results. In this paper we present further kinetic studies in viscous solvents which show a correlation of both free activation energies $\left(\Delta G^{*}\right.$ ) and diffusion coefficients $(D)$ with effective distances $R_{\text {eff }}$ associated with charge-transfer rate constants $k_{\mathrm{ct}}\left(R_{\mathrm{eff}}\right)$ for a pair of reactants, thence showing the contribution of long-range distance transfer in these rather exothermic charge-transfer interactions.

\section{Experimental Section}

The esters used, benzyl 2-naphthoate (Bz-2-Np), benzyl 9 anthroate (Bz-9-Ant), and benzyl 1-pyrenoate (Bz-1-Pn) have been synthesized from the respective acid chloride and benzyl alcohol and purified by column chromatography and further recrystallization. All compounds were identified by IR and NMR spectroscopy as reported previously. ${ }^{26}$ Triethylamine (TEA) and dimethylaniline (DMA) from B.D.H. Chemicals were destilled at reduced pressure under $\mathrm{N}_{2}$; diazabicyclo[2.2.2] octane (DAB$\mathrm{CO}$ ) and $N, N, N^{\prime}, N^{\prime}$-tetramethylphenylenediamine (TMPD) were recrystallized. Merck "Uvasol" solvents were used without further purification. The ester concentrations were in the range of $10^{-5}-10^{-4} \mathrm{~mol} \mathrm{dm}^{-3}$. Liquid paraffin "Uvasol" grade was passed through an alumina column several times until an optical density of $<0.1$ was obtained at $\lambda=275 \mathrm{~nm}$.

The viscosities of mixtures paraffin/heptane with variable ratio were determined by using a capillary viscosimeter "Technico Ubbelohde Suspended Level ASTM D445-IP71" (Gallenkamp) at $20 \pm 1{ }^{\circ} \mathrm{C}$.

The experimental details concerning the preparation of nonionic micelles of Triton-X-100 were described previously. ${ }^{25}$

Fluorescence measurements were carried out in a Perkin-Elmer MPF-3 spectrofluorimeter with a correction unit. The fluorescence decay curves were obtained by utilizing the time-correlated single-photon counting technique. ${ }^{27}$ As excitation source, a gated nanosecond flash lamp (Applied Photophysics), filled with deuterium gas and operating at $25 \mathrm{kHz}$ with a 2-ns half-width, was employed. The excitation wavelength was at $334 \mathrm{~nm}(\mathrm{Bz}-2-\mathrm{Np})$ and $361 \mathrm{~nm}$ (Bz-9-Ant, Bz-1-Pn) and the monomer fluorescence was observed at $360 \mathrm{~nm}$ (Bz-2-Np), $440 \mathrm{~nm}$ (Bz-9-Ant), and 410 $\mathrm{nm}(\mathrm{Bz}-1-\mathrm{Pn})$. Exponential decay behavior was observed in all fluorescence decay measurements over 2-3 half-lives, but small positive deviations were detected at earlier times.

\section{Results and Treatment of Data}

In this study, the fluorescence quenching of aromatic esters by aliphatic and aromatic amines was followed by steady-state and transient methods. In low-viscosity solvents (hexane and acetonitrile) linear Stern-Volmer plots were obtained by measuring either relative fluorescence intensities, $I_{0} / I$, or lifetimes, $\tau_{0} / \tau$, of the esters with amine quenchers. A typical case of quenching of $\mathrm{Bz}-1-\mathrm{Pn}$ by DMA in hexane is shown in Figure 1a.

In contrast to the behavior observed in hexane, in viscous solvents, paraffin/heptane mixtures or nonionic micelles of Triton-X-100, linear plots were observed for lifetimes $\tau_{0} / \tau$, but curve linear behavior was found for the relative fluorescence intensities $I_{0} / I$. This is illustrated in the quenching of $\mathrm{Bz}-1-\mathrm{Pn}$ by DMA

(21) V. Balzani, M. T. Indelli, M. Maestri, D. Sandrini, and F. Scandola, J. Phys. Chem., 84, 852 (1980).

(22) V. Balzani, F. Bolleta, M. T. Gandolfi, and M. Maestri, Top. Curr. Chem., 75, 1 (1978), and references cited therein.

(23) P. Siders and R. A. Marcus, J. Phys. Chem., 86, 622 (1982).

(24) S. M. B. Costa and A. L. Maçanita, J. Photochem., 11, 429 (1979).

(25) S. M. B. Costa and A. L. Maçanita, J. Phys. Chem., 84, 2408 (1980).

(26) S. M. B. Costa, A. L. Maçanita, and M. J. Prieto, J. Photochem., 11, $109(1979)$.

(27) W. R. Ware in "Creation and Detection of the Excited State", Vol. 1A, A. A. Lamola, Ed., Marcel Dekker, New York, 1971, p 213.
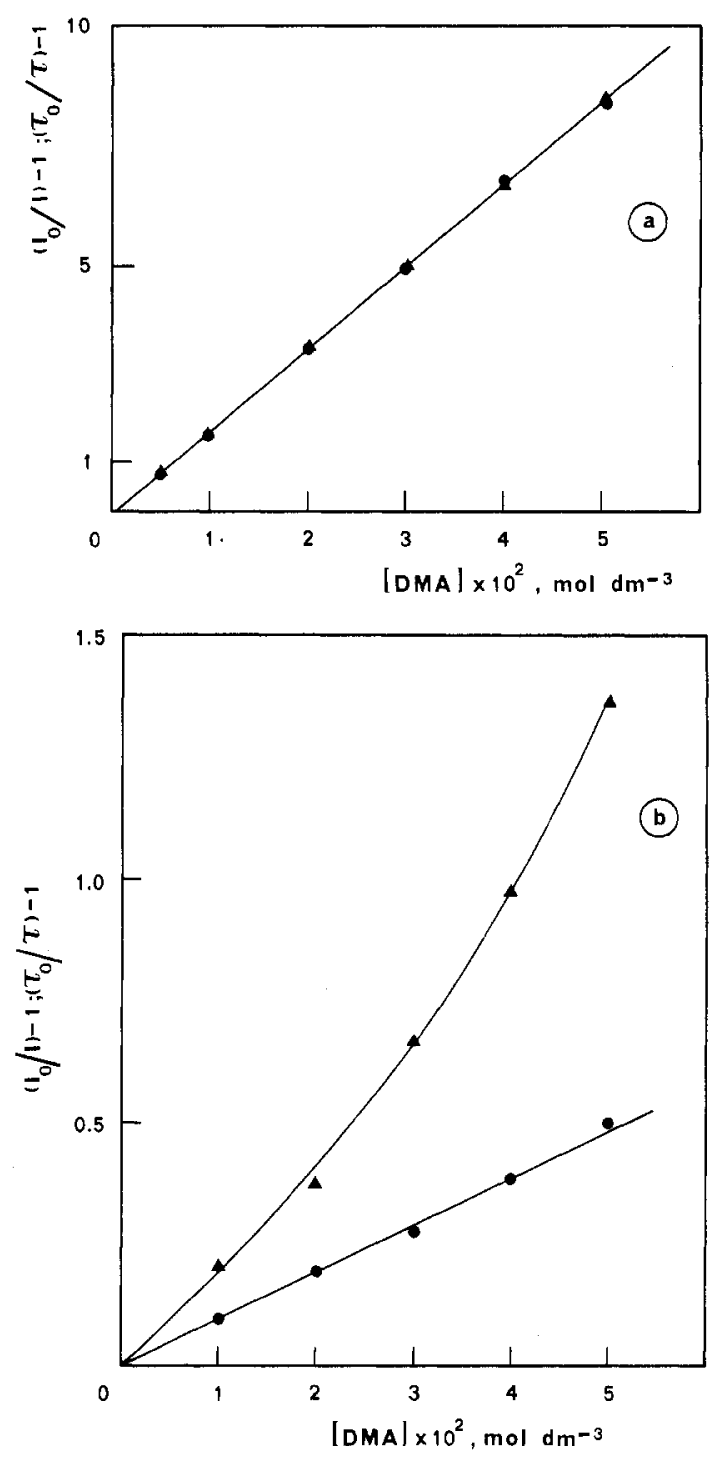

Figure 1. Fluorescence quenching of Bz-1-Pn by DMA in (a) hexane and (b) paraffin/heptane $(90: 10):(\Delta)\left(I_{0} / I\right)-1,(\bullet)\left(\tau_{0} / \tau\right)-1$.

in (90:10) paraffin/heptane shown in Figure $1 \mathrm{~b}$.

Therefore, two types of behavior are observed for these systems as the solvent viscosity is changed.

Several parameters, related to solvent properties such as the preexponential factor $k^{\circ}{ }_{\mathrm{ct}}\left(R_{\mathrm{c}}\right)$, the free activation energy $\Delta G^{\ddagger}$, diffusion coefficients $D$, and reaction distances $R^{\prime}$, were assessed from the study of same donor/acceptor systems in different media, as follows.

(A) Low-Viscosity Solvents (Hexane and Acetonitrile). The quenching observed in solvents of low viscosity can be measured by fluorescence intensities and decays in the absence and presence of quencher. Free energy changes involved in the actual charge-transfer process are easily calculated from electrochemical data since the energy of the exciplex in hexane, $\Delta G_{\left(\mathrm{A}^{-} \mathrm{Q}^{+}\right)}^{\mathrm{OHEX}}$ can be obtained by ${ }^{28}$

$$
\Delta G_{\left(\mathrm{A}^{-} \mathrm{Q}^{+}\right)}^{\circ \mathrm{HEX}_{\mathrm{D} / \mathrm{D}^{+}}}=E_{\mathrm{A} / \mathrm{A}^{-}}-E_{0-0}+0.36 \mathrm{eV}
$$

where $E_{0-0}$ is the energy of the $0-0$ transition and $E_{\mathrm{D} / \mathrm{D}^{+}}$and $E_{\mathrm{A} / \mathrm{A}^{-}}$ are the values of polarographic reduction (and oxidation) potentials determined in dimethylformamide ${ }^{29}$ and corrected for changes in solvent. ${ }^{28}$

Equation 1 was obtained from a general equation for $\Delta G^{\circ}$ which takes into account the Coulombic attractive energy at the equi-

(28) K. A. Zachariasse, Ph.D. Thesis, Amsterdam, 1972, p 22.

(29) S. M. B. Costa, A. L. Mą̧nita, E. C. C. Melo, and M. J. Prieto, J. Photochem., 11, 361 (1979). 
TABLE I: Quenching Rate Constants, $\boldsymbol{k}_{q}$, and Free Energy Changes, $\Delta G^{\circ}$

\begin{tabular}{|c|c|c|c|c|c|}
\hline & \multirow[b]{2}{*}{ system } & \multicolumn{2}{|c|}{ hexane } & \multicolumn{2}{|c|}{ acetonitrile } \\
\hline & & $\begin{array}{c}-\Delta G^{\circ} \\
\mathrm{eV}\end{array}$ & $\begin{array}{c}10^{-10} k_{\mathrm{q}}, \\
\mathrm{mol} \\
\mathrm{dm}^{-3} \mathrm{~s}^{-1}\end{array}$ & $\begin{array}{c}-\Delta G^{\circ} \\
\mathrm{eV}\end{array}$ & $\begin{array}{c}10^{-10} k_{\mathrm{q}}, \\
\mathrm{mol} \\
\mathrm{dm}^{-3} \mathrm{~s}^{-1}\end{array}$ \\
\hline 1 & $\mathrm{Bz}-1-\mathrm{Pn} / \mathrm{TMPD}^{a}$ & 1.22 & 3.06 & 1.64 & 3.04 \\
\hline 2 & Bz-9-Ant/TMPD ${ }^{a}$ & 1.15 & 2.98 & 1.57 & 3.10 \\
\hline 3 & $\mathrm{Bz}-1-\mathrm{Pn} / \mathrm{DMA}^{a}$ & 0.60 & 2.58 & 1.02 & 2.29 \\
\hline 4 & $\mathrm{Bz}-2-\mathrm{Np} / \mathrm{DABCO}^{b}$ & 0.51 & 2.57 & 0.93 & 2.30 \\
\hline 5 & $\mathrm{Bz}-1-\mathrm{Pn} / \mathrm{DABCO}{ }^{\circ}$ & 0.39 & 1.79 & 0.81 & 2.23 \\
\hline 6 & $\mathrm{Bz}-9-\mathrm{Ant} / \mathrm{DABCO}$ & 0.35 & 1.51 & 0.74 & 1.70 \\
\hline 7 & $\mathrm{Bz}-2-\mathrm{Np} / \mathrm{TEA}^{b}$ & 0.32 & 1.62 & 0.77 & 1.78 \\
\hline 8 & $\mathrm{Bz}-1-\mathrm{Pn} / \mathrm{TEA}^{b}$ & 0.23 & 1.13 & 0.65 & 1.22 \\
\hline 9 & Bz-9-Ant/TEA ${ }^{b}$ & 0.16 & 1.07 & 0.58 & 1.88 \\
\hline
\end{tabular}

${ }^{a}$ This work. ${ }^{b}$ Reference 24 .

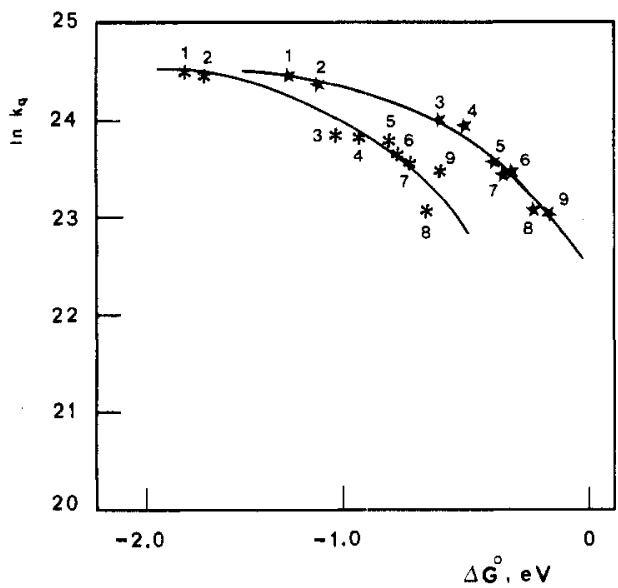

Figure 2. Variation of $\ln k_{\mathrm{q}}$ with $\Delta G^{\circ}$ in ( $\star$ ) hexane and $\left(^{*}\right)$ acetonitrile: (1) Bz-1-Pn/TMPD, (2) Bz-9-Ant/TMPD, (3) Bz-1-Pn/DMA, (4) Bz-2-Np/DABCO, (5) Bz-1-Pn/DABCO, (6) Bz-9-Ant/DABCO, (7) Bz-2-Np/TEA, (8) Bz-1-Pn/TEA, (9) Bz-9-Ant/TEA.

librium distance, the solvation energy of the exciplex, and the resonance energy. ${ }^{30}$ The latter which includes a destabilizing term $U_{\text {dest }}$ and a stabilizing term $U_{\text {stab }}$ was made equal to zero, which is approximately valid in charge-transfer exciplexes. ${ }^{31}$ The standard free energy change of the charge-transfer step $\Delta G^{\circ}{ }_{\text {ct }}$ was assumed to be equal to the standard free energy change of the overall net charge-transfer reaction since work terms- $\omega_{r}$ required to bring the reactants together and $\omega_{p}$ the corresponding one for the products-were neglected in this case because the species involved are neutral. ${ }^{10}$

In the same way eq $2^{28}$ was used for a polar solvent such as acetonitrile.

$$
\Delta G_{\left({ }^{2} \mathrm{~A}^{-} \ldots \mathrm{Q}^{+}\right)}^{\circ \mathrm{MEC}}=E_{\mathrm{D} / \mathrm{D}^{+}}-E_{\mathrm{A} / \mathrm{A}^{-}}-E_{0-0}-0.06 \mathrm{eV}
$$

Table I presents values of $k_{\mathrm{g}}$ and $\Delta G^{\circ}$ obtained in hexane and acetonitrile. The variation of $\log k_{\mathrm{q}}$ vs. $\Delta G^{\circ}$ in these solvents is the one expected in charge-transfer processes, and a plateau is reached for rather negative values of $\Delta G^{\circ}$ (Figure 2).

The values of $k_{\mathrm{q}}$ obtained in all systems only vary by a factor of 3 and are near the diffusion-controlled limit. Therefore, the energetics are better stressed with the unimolecular rate constant associated with the charge-transfer step, $k_{\mathrm{ct}}$.

The interaction of singlet excited states of these aromatic esters with tertiary amines is of charge-transfer nature as shown by the detection of radical ions of esters in polar solvents and from the large dipolar moments of exciplexes observed in nonpolar solvents. ${ }^{29}$

In either case, the formation of an exciplex or a radical ion pair is thought to proceed from an encounter complex. The kinetics

(30) H. Knibbe, Ph.D. Thesis, Amsterdam, 1970

(31) A. Weller in "The Exciplex", M. Gordon and W. R. Ware, Eds., Academic Press, New York, 1975, p 23 of these processes are described by

Scheme I

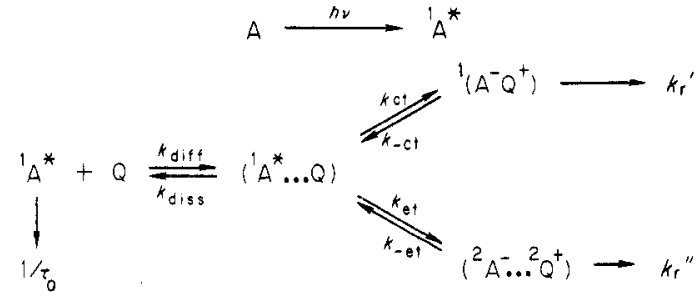

where $k_{\text {diff }}$ and $k_{\text {diss }}$ are rate constants for diffusion-controlled formation and dissociation of the encounter complex ( ${ }^{1} \mathrm{~A}^{*}$...Q $)$, respectively; $k_{\mathrm{ct}}$ and $k_{\mathrm{et}}$ are rate constants for the charge or electron transfer from the encounter, and $k_{-c t}$ and $k_{-e t}$ are rate constants for the back-transfer; $k_{\mathrm{r}}^{\prime}$ and $k_{\mathrm{r}}^{\prime \prime}$ are the sum of all the deactivation rate constants (other than back-transfer) for the exciplex and radical-ion pair, respectively, and $\tau_{0}$ is the lifetime of the monomer.

The reversible formation of the exciplex or radical ion pair makes it difficult to obtain $k_{\mathrm{ct}}$ and $k_{\text {-ct }}$ directly. Therefore, most of the information available on such processes is based upon $k_{\mathrm{q}}$, which is easily obtainable from steady-state experiments.

The reversibility of charge transfer in these systems is negligible in the range of $153-333 \mathrm{~K}$ as was demonstrated by the temperature dependence of the excited complex formation. ${ }^{24}$ Thus, the overall bimolecular quenching rate constant $k_{\mathrm{q}}$ correlates with the other rate constants by eq 3 , assuming that only one of the two possible pathways-exciplex or ion-pair formation-is occurring.

$$
k_{\mathrm{q}}=k_{\mathrm{diff}} k_{\mathrm{ct}} /\left(k_{\mathrm{ct}}+k_{\mathrm{diss}}\right)=\gamma k_{\mathrm{diff}}
$$

where $\gamma$ is the charge-transfer efficiency from the encounter complex ${ }^{24}$ and $k_{\text {diff }}$ and $k_{\text {diss }}$ can be calculated by eq $4^{32}$ and $5,^{33,34}$

$$
\begin{gathered}
k_{\text {diff }}=4 \pi N^{\prime} R_{\mathrm{c}} D \\
k_{\text {diss }}=3 D R_{\mathrm{c}}^{-2}
\end{gathered}
$$

In eq 4 and $5 R_{\mathrm{c}}$ is the collisional distance taken as the sum of van der Waals radii; $D$ is the sum of individual diffusion coefficients for the donor and the acceptor; ${ }^{29} N^{\prime}=$ Avogadro's number $N \times 10^{-3}$. In eq 5 it is assumed that the binding energy of the encounter complex is zero.

The unimolecular charge-transfer rate constant $k_{\text {ct }}$ can be evaluated by ${ }^{24}$

$$
k_{\mathrm{ct}}=\gamma /(1-\gamma) k_{\mathrm{diss}}
$$

This relation assumes that charge transfer takes place at collisional distances but with efficiencies lower than unity. Therefore, it is only applicable to nondiffusion-controlled processes $\left(k_{\mathrm{q}}<k_{\text {diff }}\right)$ where, at the collisional distance $R_{\mathrm{c}}$, the magnitude of the encounter complex dissociation rate constant $k_{\text {diss }}$ is significant as compared with the charge-transfer rate constant $k_{\mathrm{ct}}$.

Table II presents experimental data obtained from these systems. The free energies of activation $\Delta G_{s}{ }^{*}$ were obtained from Weller's empirical eq 7 , adequate for very exothermic reactions ${ }^{7 b}$

$$
\Delta G^{\ddagger}=\left[\left(\Delta G^{\circ} / 2\right)^{2}+(\lambda / 4)^{2}\right]^{1 / 2}+\Delta G^{\circ} / 2
$$

where $\lambda$ is the solvent repolarization energy, and with eq $8^{9}$

$$
\ln k_{\mathrm{ct}}=\ln k_{\mathrm{ct}}^{\circ}\left(R_{\mathrm{c}}\right)-\Delta G^{\ddagger} / k_{\mathrm{B}} T
$$

A value of $\lambda=0.64 \mathrm{eV}$ (in hexane) gave the best linear fit for eq 8 (slope $1 / k_{\mathrm{B}} T=38.8 \mathrm{eV}^{-1} ; T=293 \mathrm{~K}$ ) as shown in Figure 3. The intercept ( $\left.\ln k_{\mathrm{ct}}\left(R_{\mathrm{c}}\right)=26.64\right)$ gives the preexponential factor at the collisional distance, $k^{\circ}{ }_{\mathrm{ct}}\left(R_{\mathrm{c}}\right)=3.7 \times 10^{11} \mathrm{~s}^{-1}$. If one uses Agmon and Levine's equation ${ }^{35}$ derived on the basis of a thermodynamic treatment of concerted reaction kinetics, the results are identical. In acetonitrile the lack of experimental data

(32) A. H. Alwattar, M. D. Lumb, and J. B. Birks in "Organic Molecular Photophysics", Vol. I, J. B. Birks, Ed., Wiley-Interscience, 1973, p 403.

(33) C. Lewis and W. R. Ware, Mol, Photochem., 5, 477 (1973).

(34) W. R. Ware and J. Novros, J. Phys. Chem., 70, 3246 (1966)

(35) N. Agmon and R. D. Levine, Chem. Phys. Lett., 52, 197 (1977). 
TABLE II: Quenching Efficiencies, $\gamma$, Charge-Transfer Rate Constants, $k_{\mathrm{ct}}$, and Free Energies of Activation, $\Delta G^{*}$

\begin{tabular}{|c|c|c|c|c|c|c|c|}
\hline & \multirow[b]{2}{*}{ system } & \multicolumn{3}{|c|}{ hexane } & \multicolumn{3}{|c|}{ acetonitrile } \\
\hline & & $\gamma$ & $10^{-10} k_{\mathrm{ct}}, \mathrm{s}^{-1}$ & $\Delta G^{\ddagger}, \mathrm{eV}$ & $\gamma$ & $10^{-10} k_{\mathrm{ct}}, \mathrm{s}^{-1}$ & $\overline{\Delta G^{*}, \mathrm{eV}}$ \\
\hline 1 & $\mathrm{Bz}-1-\mathrm{Pn} / \mathrm{TMPD}^{a}$ & 0.82 & 17.9 & 0.021 & $c$ & $c$ & 0.043 \\
\hline 2 & Bz-9-Ant/TMPD ${ }^{a}$ & 0.79 & 15.2 & 0.022 & $c$ & $c$ & 0.045 \\
\hline 3 & $\mathrm{Bz}-1-\mathrm{Pn} / \mathrm{DMA}^{a}$ & 0.64 & 7.6 & 0.040 & 0.77 & 10.3 & 0.067 \\
\hline 4 & $\mathrm{Bz}-2-\mathrm{Np} / \mathrm{DABCO}{ }^{b}$ & 0.66 & 7.4 & 0.046 & 0.76 & 10.9 & 0.073 \\
\hline 5 & $\mathrm{Bz}-1-\mathrm{Pn} / \mathrm{DABCO}{ }^{a}$ & 0.48 & 3.6 & 0.057 & 0.75 & 9.18 & 0.082 \\
\hline 6 & 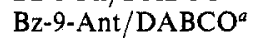 & 0.40 & 3.0 & 0.066 & 0.62 & 5.9 & 0.088 \\
\hline 7 & $\mathrm{Bz}-2-\mathrm{Np} / \mathrm{TEA}^{b}$ & 0.43 & 3.0 & 0.062 & 0.59 & 5.3 & 0.085 \\
\hline 8 & $\mathrm{Bz}-1-\mathrm{Pn} / \mathrm{TEA}^{b}$ & 0.31 & 1.8 & 0.082 & 0.42 & 2.16 & 0.098 \\
\hline 9 & Bz-9-Ant/TEA $b$ & 0.29 & 1.6 & 0.099 & 0.61 & 1.96 & 0.106 \\
\hline
\end{tabular}

${ }^{a}$ This work. ${ }^{b}$ Reference 24 . ${ }^{c}$ Diffusion-controlled limit.

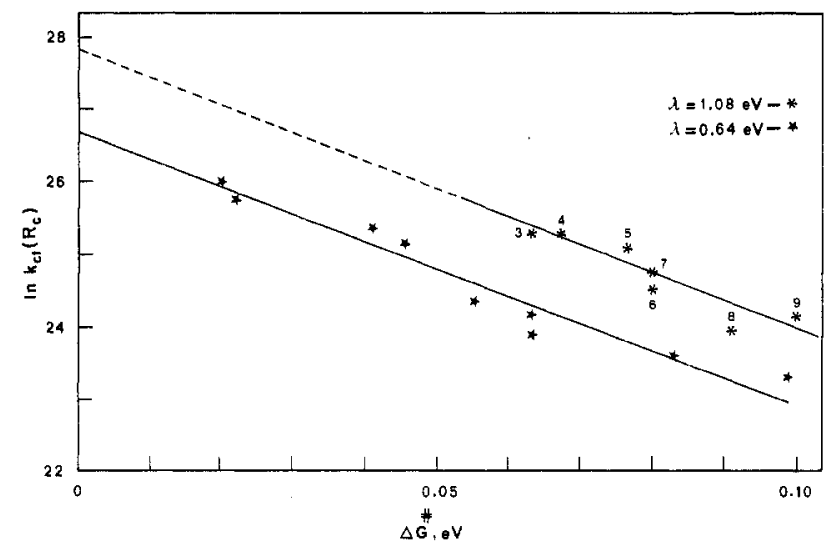

Figure 3. Dependence of $\ln k_{\mathrm{ct}}$ on $\Delta G^{*}$ in $(\star)$ hexane and $\left(^{*}\right)$ acetonitrile: (1) Bz-1-Pn/TMPD, (2) Bz-9-Ant/TMPD, (3) Bz-1-Pn/DMA, (4) $\mathrm{Bz}-2-\mathrm{Np} / \mathrm{DABCO}$, (5) Bz-1-Pn/DABCO, (6) Bz-9-Ant/DABCO, (7) Bz-2-Np/TEA, (8) Bz-1-Pn/TEA, (9) Bz-9-Ant/TEA.

for systems with more negative values only allows an extrapolation of $\ln k^{\circ}{ }_{\mathrm{ct}}\left(R_{\mathrm{c}}\right)=27.8\left(k^{\circ}{ }_{\mathrm{ct}}\left(R_{\mathrm{c}}\right)=1.2 \times 10^{12} \mathrm{~s}^{-1}\right)$ and $\lambda=1.08$ $\mathrm{eV}$ (Figure 3).

(B) Viscous Solvents (Liquid Paraffin/Heptane Mixtures). The effect of diffusion phenomena on the quenching process was investigated by studying the fluorescence quenching of one of the systems Bz-1-Pn/DMA in mixtures of liquid paraffin/heptane with variable composition to enable a gradual decrease of the diffusion coefficient (increase of viscosity).

An exciplex emission was also observed in these mixtures ( $\lambda$ $=540 \mathrm{~nm}$ ) similarly to that detected in pure hexane.

The quenching rate constants obtained from $\left(\tau_{0} / \tau\right)-1$ vs. [Q] are of the order of diffusion-controlled rate constants $\left(k_{\mathrm{q}} \simeq k_{\text {diff }}\right)$. As the solvent viscosity increases, $\left(I_{0} / I\right)-1$ vs. [Q] deviates from $\left(\tau_{0} / \tau\right)-1$ vs. [Q] progressively, and the curvature in the former plots is more pronounced (Figure 4). This effect cannot be attributed to ground-state association since there is no evidence for it in the absorption spectra even at very high concentrations, and the equilibrium constant calculated by assuming such an effect is negative. In such cases $k_{\mathrm{ct}}$ can no longer be determined by eq 6 and Scheme I must be replaced by

Scheme II

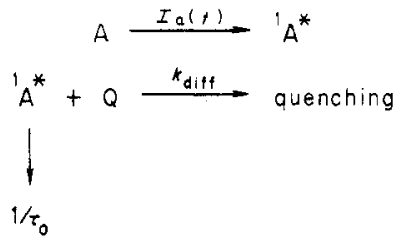

The data obtained (Figure 4) were treated with the following equations:

$$
\begin{gathered}
\tau_{0} / \tau=1+k_{\mathrm{q}} \tau_{0}[\mathrm{Q}] \quad\left(k_{\mathrm{q}}=4 \pi N^{\prime} R^{\prime} D\right) \\
I_{0} / I=\left(1+k_{\mathrm{q}} \tau_{0}[\mathrm{Q}]\right) Y^{-1} e^{\left.N^{\prime} \gamma \mathrm{Q}\right]} \\
\ln \left[\left(I_{0} / I\right) Y /\left(\tau_{0} / \tau\right)\right]=N^{\prime} \mid V[\mathrm{Q}]
\end{gathered}
$$
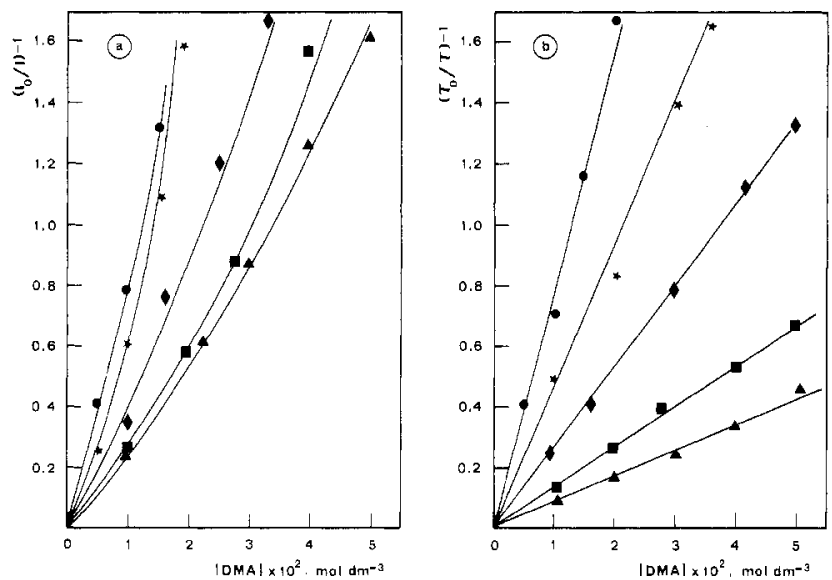

Figure 4. The effect of viscosity in the fluorescence quenching of Bz-1-Pn by DMA in paraffin/heptane mixtures: (a) steady-state $\left(I_{0} / D\right)-1$, (b) transient state $\left(\tau_{0} / \tau\right)-1$. The paraffin/heptane ratios are the following:

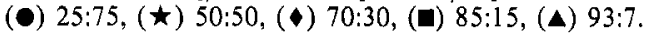

TABLE III: Diffusion Coefficients, $D$, Effective Distances of Encounter, $R^{\prime}$, and Viscosities, $\eta$, of the System Bz-1-Pn/DMA in Paraffin/Heptane (P:H)

\begin{tabular}{llccc}
\hline \multicolumn{1}{c}{$\mathrm{P}: \mathrm{H}$} & $\eta, \mathrm{cP}$ & $\begin{array}{c}10^{13} R^{\prime} \mathrm{D}^{a} \\
\mathrm{~cm}^{3} \mathrm{~s}^{-1}\end{array}$ & $\begin{array}{c}10^{6} D, \\
\mathrm{~cm}^{2} \mathrm{~s}^{-1}\end{array}$ & $10^{8} R^{\prime}, \mathrm{cm}$ \\
\hline $93: 7$ & 34 & 0.84 & 0.7 & $12.0 \pm 0.9$ \\
$90: 10$ & 20 & 1.37 & 1.2 & $10.3 \pm 0.9$ \\
$85: 15$ & 15 & 1.84 & 1.6 & $10.7 \pm 0.9$ \\
$80: 20$ & 10 & 2.08 & 2.3 & $9.1 \pm 0.9$ \\
$70: 30$ & 6.2 & 3.8 & 4.0 & $9.5 \pm 0.9$ \\
$50: 50$ & 3.6 & 6.3 & 7.5 & $8.3 \pm 0.9$ \\
$25: 75$ & 1.25 & 13 & 18 & $7.3 \pm 0.3$ \\
cyclohexane & 0.98 & & 22 & $7.3 \pm 0.3^{b}$ \\
hexane & 0.31 & & 66 & $7.3 \pm 0.3^{b}$
\end{tabular}

${ }^{a}$ Taken from Figure $4 \mathrm{~b} .{ }^{b}$ Collisional distance, $R_{\mathrm{c}}$.

where the term $Y$ accounts for the so-called transient effect observed in the experimental fluorescence steady-state measurements (Figure $4 a$ ) and $V$ is the volume limited by the reactional and collisional spheres, given by

$$
V=4 / 3 \pi\left(R^{\prime 3}-R_{\mathrm{c}}{ }^{3}\right)
$$

The additional term $\exp (N \mathcal{V}[\mathrm{Q}])$ accounts for the instantaneous deactivation of ${ }^{1} \mathrm{~A}^{*}$ by quencher molecules placed on a spherical shell of volume $V .{ }^{14}$ The analysis of the data involves a system of two equations, (9) and (10), with two unknowns $\left(R^{\prime}\right.$ and $\left.D\right)$.

The product $R^{\prime} D$ shown in Table III can be extracted from the Stern-Volmer plots obtained, $\left(\tau_{0} / \tau\right)-1$ vs. [Q]. If $R^{\prime}$ is arbitrarily chosen, $D$ can be calculated and $Y\left(R^{\prime} D\right)$ determined (see Appendix). The values of $I_{0} / I$ can then be calculated (eq 10) and compared with the experimental data. The procedure is repeated until agreement between calculated and experimental values is obtained.

Further checking of this iterative method has been made using eq 11. Indeed, a linear relationship of $\ln \left[\left(I_{0} / I\right) Y /\left(\tau_{0} / \tau\right)\right]$ vs. [Q] is obtained only with the correct value of $R^{\prime}$ taken from the 


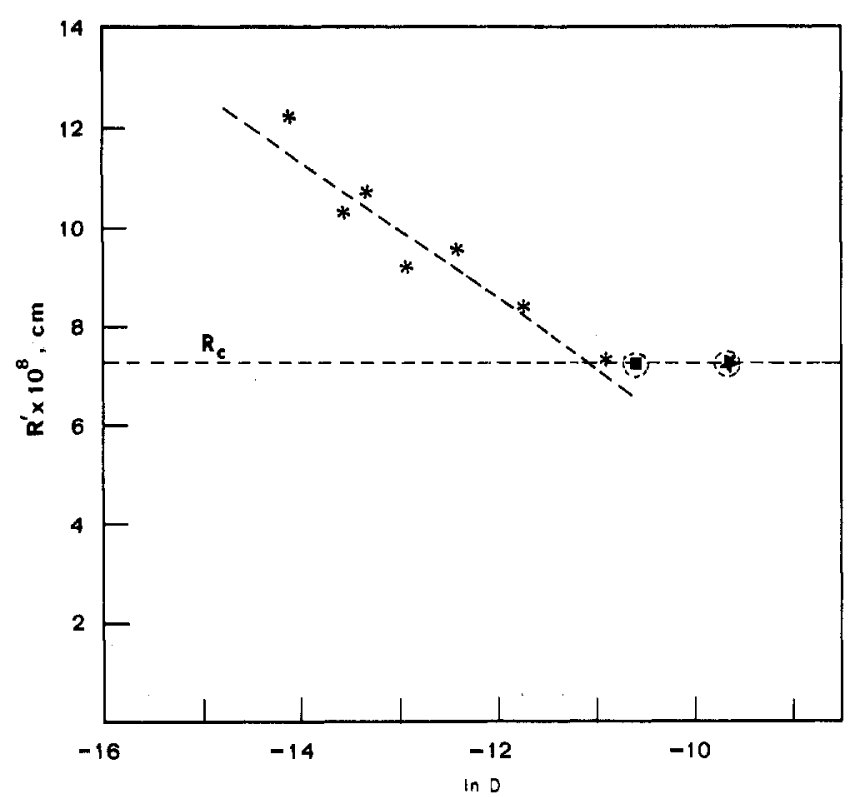

Figure 5. Variation of $R^{\prime}$ with $\ln D$ in the system Bz-1-Pn/DMA with several mixtures of paraffin/heptane $\left({ }^{*}\right)$, (circled box) cyclohexane, and (circled star) hexane (at collisional distances, $R_{\mathrm{c}}$ ).

combination of eq 11 and 12 . The collisional distance, $R_{\mathrm{c}}=7.3$ $\times 10^{-8} \mathrm{~cm}$, was assumed to be the sum of van der Waals radii, $R^{\mathrm{vw}}$ (calculated by Edward's method ${ }^{36}$ ), of species $R_{\mathrm{Bz}-1 \cdot \mathrm{Pn}}^{\mathrm{vw}}=4.15$ $\times 10^{-8} \mathrm{~cm}$ and $R_{\mathrm{DMA}}^{\mathrm{vW}}=3.12 \times 10^{-8} \mathrm{~cm}$ of acceptor and donor molecules.

Table III presents values of $R^{\prime}$ and $D$ estimated for several mixtures, as well as viscosities determined experimentally.

The variation of $R^{\prime}$ with $\ln D$ follows a rather good linear correlation for systems with $D<1.8 \times 10^{-5} \mathrm{~cm}^{2} \mathrm{~s}^{-1}$ as shown in Figure 5. The points indicated for cyclohexane and hexane, if one assumes that charge transfer in these solvents takes place at collisional distances, fall off this line as expected.

\section{Discussion}

The data presented in the previous section, concerning charge-transfer processes in a family of redox partners, show essentially two features:

(A) In nonviscous solvents the partners interact at collisional distances, and the charge-transfer rate constant, $k_{\mathrm{ct}}$, can be determined on the basis of the encounter complex scheme approach.

(B) In viscous solvents, reactional distances estimated from experimental data are larger than the collisional ones and increase with the solvent viscosity included in diffusion coefficients $D$.

In order to discuss the results obtained in this work, it is necessary to analyze the data under the current theories of electron or charge transfer.

The rate constant for charge transfer in its general form is given by Marcus's equation ${ }^{11}$

$$
k_{\mathrm{ct}}=\left(k_{\mathrm{B}} T / h\right)\langle\kappa\rangle \exp \left(-\Delta G^{*} / k_{\mathrm{B}} T\right)
$$

where $\langle\kappa\rangle$ is the nonadiabaticity factor related to the probability of charge transfer in the activated complex. Adiabatic reactions have $\langle\kappa\rangle$ constant, and eq 13 is very adequate for endothermic and moderately exothermic reactions.

For a nonadiabatic transfer in the absence of nuclear tunneling, the factor $\langle k\rangle$ is given by ${ }^{37,38}$

$$
\langle\kappa\rangle=2\left[\pi^{3} /\left(\lambda\left(k_{\mathrm{B}} T\right)^{3}\right)\right]^{1 / 2} V(R)^{2}
$$

where the electronic matrix element $V(R)$ is given by ${ }^{39}$

(36) J. T. Edward, J. Chem. Educ., 47, 261 (1970).

(37) N. Sutin in "Tunneling in Biological Systems", B. Chance, D. C. Devault, H. Frauenfelder, J. R. Schrieffer, and N. Sutin, Eds., Academic Press, New York, 1979, p 201.

(38) B. S. Brunschwig, J. Logan, M. D. Newton, and N. Sutin, J. Am. Chem. Soc., 102, 5798 (1980).

$$
V(R)=V\left(R_{\mathrm{c}}\right) \exp \left[-\left(R-R_{\mathrm{c}}\right) / L\right]
$$

$R$ being the center-to-center separation distance, $\left(R-R_{\mathrm{c}}\right)$ the edge-to-edge distance, and $L$ the average radii of the donor and acceptor orbitals.

Equations 13,14 , and 15 can be combined to give a general expression (eq 16) for a distance-dependent charge-transfer rate constant $k_{\mathrm{ct}}(R)$

$k_{\mathrm{ct}}(R)=k_{\mathrm{ct}}^{\circ}\left(R_{\mathrm{c}}\right) \exp \left[-2\left(R-R_{\mathrm{c}}\right) / L\right] \exp \left(-\Delta G^{\ddagger} / k_{\mathrm{B}} T\right)$

with

$$
k^{\circ}{ }_{\mathrm{ct}}\left(R_{\mathrm{c}}\right)=(2 \pi / \hbar)\left(4 \pi \lambda k_{\mathrm{B}} T\right)^{-1 / 2} V\left(R_{\mathrm{c}}\right)^{2}
$$

At collisional distances eq 16 reduces to the well-known eq 8 which was used to treat results presented in section $3 \mathrm{~A}$.

The data obtained does not show the decrease of the rate constant expected from Marcus theory in the abnormal region and is in good agreement with Rehm-Weller's results.

Indeed, if any inflection exists in the $\ln k_{\mathrm{ct}}$ vs. $\Delta G^{\ddagger}$ plot, it is not directly accessible from our studies in solution due to the diffusion limit. On the other hand, quantum mechanics theory predicts that the abnormal region should be reached at more negative $\Delta G^{\circ}{ }_{\mathrm{s}}$ values than those expected from Marcus theory, namely for molecules having a large number of vibrational modes, as it is the case for aromatic molecules. Thus, it is possible that our "best" system is not exothermic enough to observe the decrease of the charge-transfer rate constants.

Under these conditions, when a classical approach is used, eq 7 seemed to be a reasonable choice to calculate $\Delta G^{*}$ from $\Delta G^{\circ}$.

The value of repolarization energy in acetonitrile $(\lambda=1.08 \mathrm{eV})$ is in good agreement with the expected theoretical value in polar solvents $(\lambda=1.10 \mathrm{eV})$ but is higher than some found in the literature. $^{7 a}$ It is possible that in the aromatic esters/aliphatic amines conformational changes of the reactants required for the electron transfer may contribute to a higher value of $\lambda$. Indeed, Balzani $^{40}$ and De Schryver ${ }^{41}$ have also found a value of $\lambda=0.90$ $\mathrm{eV}$ using aliphatic amines as donors. In a nonpolar solvent, the contribution due to the reorganization of the solvent is negligible and therefore the value of $\lambda$ in hexane should mainly reflect the reorganization of the reactants, but there are no terms of comparison in the literature. The charge transfer in this solvent occurs via the exciplex and consequently is more stringent as regards the nuclear configuration of the reactants. The value of the parameter $\lambda$ may be associated with the modification of the internuclear distance and bond angles required for the exciplex formation such as rehybridization of the nitrogen atom in amines.

The preexponential factor obtained $\left(k^{\circ}{ }_{\mathrm{ct}}\left(R_{\mathrm{c}}\right)=3.7 \times 10^{11} \mathrm{~s}^{-1}\right)$ cannot be discussed as such since a collisional distance is implicit therein. However, it is interesting to note that with this parameter and $\lambda=0.64 \mathrm{eV}$ a value for the matrix electronic element $V\left(R_{\mathrm{c}}\right)$ $\simeq 10^{-3} \mathrm{eV}\left(\simeq 30 \mathrm{~cm}^{-1}\right)$ can be obtained. This estimation seems to indicate a weaker interaction than that expected in adiabatic reactions $\left(V\left(R_{\mathrm{c}}\right)>100 \mathrm{~cm}^{-1}\right)$ and might mean that the reaction is nonadiabatic at collisional and larger distances as one would expect from the exothermicity observed in these systems. However, the constant value of the preexponential factor observed throughout the whole family system shows that these systems, if nonadiabatic, are equally nonadiabatic and can be treated within the context of Marcus theory. ${ }^{42}$.

The data obtained in section 3B may be discussed on the basis of a formalism adequate to diffusion-controlled reactions in liquid phase. These include long-range interactions which are distance dependent. Pilling and Rice ${ }^{176}$ examined the effect of a reaction probability which falls off exponentially with the distance, by solving the diffusion equation

(39) A. Namiki, N. Nakashima, K. Yoshihara, Y. Ito, and T. Higashimura, J. Phys. Chem. 82, 1901 (1978).

(40) R. Ballardini, G. Vallini, M. T. Indelli, F. Scandola, and V. Balzani, J. Am. Chem. Soc., 100, 7219 (1978).

(41) M. Van der Auweraer, A. Gilbert, and F, C. De Schryver, J. Am. chem. Soc., 102, 4002 (1980).

(42) J. C. W. Chien, J. Phys. Chem., 82, 2158 (1978). 


$$
\frac{\partial S(r, t)}{\partial t}=\frac{D}{r} \frac{\partial^{2} S(r, t) r}{\partial r^{2}}-A \exp (-2 r / L) S(r, t)
$$

where $S(r, t)$ is the time- and distance-dependent average concentration of acceptor and donor, $D$ is the coefficient of relative diffusion, $r$ is the distance between the central donor and acceptor, and the sink term $[A \exp (-2 r / L)]$ can either imply an exchange interaction or an electron-transfer process. The physical meaning of the frequency factor $A$ (equivalent to the rate at "zero" distance) will vary accordingly.

Equation 18 was solved for a steady-state concentration profile (infinite time) with a rate constant $k_{\mathrm{q}}$

$$
k_{\mathrm{q}}(t \rightarrow \infty)=4 \pi N^{\prime} R_{\mathrm{eff}} D
$$

where $R_{\text {eff }}$ is the effective encounter distance

Pilling et al. ${ }^{16 \mathrm{~b}}$ have shown that for small values of diffusion coefficients $\left(D<4.0 \times 10^{-6} \mathrm{~cm}^{2} \mathrm{~s}^{-1}\right)$ it is valid to use eq 20 to calculate $R_{\text {eff }}$,

$$
R_{\mathrm{eff}}=(L / 2)\left[2 E+\ln \left(A L^{2} /(4 D)\right)\right]
$$

with the Euler's constant $E=0.57721$.

Equation 20 implies that transfer at long distances dominates and predicts that $R_{\text {eff }}$ is independent of $R_{\mathrm{c}}$ increasing with the decrease of the diffusion coefficient. by

The frequency factor of an activated charge transfer is given

$$
A=k_{0} \exp \left(-\Delta G^{*} / k_{\mathrm{B}} T\right)
$$

with

$$
k_{0}=k^{\circ}{ }_{\mathrm{ct}}\left(R_{\mathrm{c}}\right) \exp \left(+2 R_{\mathrm{c}} / L\right)
$$

Substitution of eq 21 into eq 20 leads to

$$
R_{\text {eff }}=(L / 2) \ln \left[k_{0}\left(L e^{E} / 2\right)^{2}\right]-(L / 2)\left(\Delta G^{*} / k_{\mathrm{B}} T+\ln D\right)
$$

Whereas this equation is based on a model which implies a continuous electron transfer step, in contrast with Ware's model adopted in the kinetic analysis presented in this paper which assumes a discontinuous electron-transfer probability, it may nevertheless be interesting to apply eq 23 to our results (Figure 5) since eq 23 also predicts a linear correlation of $R_{\text {eff }}$ with $\ln D$ (if $\Delta G^{*}$ is kept constant).

For instance, the same system (Bz-1-Pn/DMA) was studied in several solvent mixtures with the same polarity and variable viscosities. In such conditions, even though the refractive index $n$ of each mixture is different and consequently the polarizability also changes, it may be valid to assume that $\Delta H^{*}$ salues are not much affected and consequently $\Delta G^{*}$ will remain constant.

The identification of the linear correlation shown in Figure 5 with eq 23 gives from the slope $(L / 2)$ a value of $L \simeq(2.7 \pm 0.2)$ $\times 10^{-8} \mathrm{~cm}$ for this donor-acceptor pair. The intercept at the origin $\left\{(L / 2)\left[\ln \left(k_{0} L^{2} e^{2 E} / 4\right)-\left(\Delta G^{*} / k_{\mathrm{B}} T\right)\right]\right\}$ is -7.337 . Thus, if $E=$ $0.577, L=2.7 \times 10^{-8} \mathrm{~cm}$, and $\Delta G^{*}(\mathrm{Bz}-1-\mathrm{Pn} / \mathrm{DMA})=0.04 \mathrm{~V}$, a value of $k_{0}=(4.5 \pm 1) \times 10^{13} \mathrm{~s}^{-1}$ is obtained.

We have previously investigated the same systems in nonionic micelles of Triton X-100, and the quenching was attributed to a charge-transfer diffusion-controlled process. ${ }^{22}$ It was assumed that the quenching was dynamic and occurred inside the micellar phase.

The free energy changes $\Delta G^{\circ}$ in this medium were evaluated by eq 24 , obtained by assuming a medium polarity around the probes similar to diethyl ether $(\epsilon=10),{ }^{43,44}$

$$
\Delta G^{\circ}=E_{\mathrm{D} / \mathrm{D}^{+}}-E_{\mathrm{A} / \mathrm{A}^{-}}-E_{0-0}+0.1 \mathrm{eV}
$$

The repolarization energy $\lambda=1.0 \mathrm{eV}$ was used to calculate $\Delta G^{*}$. $R^{\prime}$ values obtained in this medium ${ }^{22}$ correlate with $\Delta G^{*}$ as illustrated in Figure 6, and a reasonable linear plot is also observed.

(43) A. L. Maçanita, Ph.D. Thesis, Lisboa, 1981.

(44) E. C. C. Melo, unpublished results.

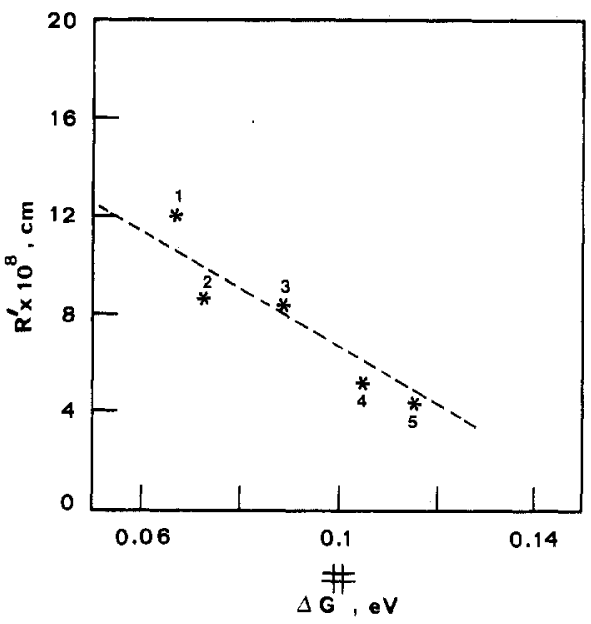

Figure 6. Variation of $R^{\prime}$ with $\Delta G^{*}$ in Triton X-100 micelles (*): (1) Bz-1-Pn/DMA, (2) Bz-9-Ant/DMA, (3) Bz-2-Np/TEA, (4) Bz-1Pn/TEA, (5) Bz-9-Ant/TEA.

TABLE IV: Estimated Charge-Transfer Rate Constants, $\boldsymbol{k}_{\mathrm{et}}\left(\boldsymbol{R}_{\mathrm{eff}}\right)$

\begin{tabular}{ccccc}
\hline $\begin{array}{c}10^{8} R_{\text {eff }}{ }^{a} \\
\mathrm{~cm}\end{array}$ & $\begin{array}{c}10^{6} D, \\
\mathrm{~cm}^{2} \mathrm{~s}^{-1}\end{array}$ & $\begin{array}{c}10^{-9} k_{\mathrm{ct}}\left(R_{\text {eff }}\right), \\
\mathrm{s}^{-1}\end{array}$ & $\begin{array}{c}10^{-9} k_{\mathrm{ct}}^{\prime}\left(R_{\text {eff }}\right){ }^{c} \\
\mathrm{~s}^{-1}\end{array}$ & $f^{d}$ \\
\hline 12.0 & 0.7 & 2.2 & 1.2 & 1.8 \\
10.7 & 1.6 & 5.4 & 2.8 & 1.9 \\
9.5 & 4 & 14.6 & 7.9 & 2.0
\end{tabular}

${ }^{a} R^{\prime}$ was replaced by $R_{\text {eff. }}{ }^{b}$ Equation $16 .{ }^{c}$ Equation $23 .{ }^{d} f=k_{\text {ct- }}$ $\left(R_{\text {eff }}\right) / k_{\text {ct }}^{\prime}\left(R_{\text {eff }}\right)$.

An effective diffusion coefficient $D=1.5 \times 10^{-6} \mathrm{~cm}^{2} \mathrm{~s}^{-1}$ was estimated for this medium, and therefore since $D$ is constant, a linear correlation of $R_{\text {eff }}$ with $\Delta G^{*}$ is predicted. If again the linear plot shown in Figure 6 is identified with eq 23, the slope $\left(L / 2 k_{\mathrm{B}} T\right)$ and intercept $\left\{(L / 2)\left[\ln \left(k_{0} L^{2} e^{2 E} / 4 D\right)\right]\right\}$ enable the estimation of parameters $L=(2.8 \pm 0.2) \times 10^{-8} \mathrm{~cm}$ and $k_{0}=(3.0 \pm 1) \times 10^{13}$ $\mathrm{s}^{-1}$.

A comparison with data taken from the studies reported in nonviscous solvents such as hexane is also relevant. If the same value of $L$ is used in hexane $\left(L=(2.7 \pm 0.2) \times 10^{-8} \mathrm{~cm}\right)$ and with $R_{\mathrm{c}}=7.3 \times 10^{-8} \mathrm{~cm}$, it is possible to obtain from the preexponential factor at collisional distance $k^{\circ}{ }_{\mathrm{ct}}\left(R_{\mathrm{c}}\right)$ the value of $k_{0}=(0.9 \pm$ $0.3) \times 10^{14} \mathrm{~s}^{-1}$ (eq 22) in hexane. The Bohr radius $L$ should vary with the polarity, and therefore it is not possible to calculate $k_{0}$ in acetonitrile directly from eq 22 .

While large experimental errors are involved, the similarity of orders of magnitude of the "true" preexponential factor $k_{0}$ ("zero" distance) obtained from different experiments seems to enable one to compare data from these two models.

The approximation contained in eq 20 is valid when the transfer rate at long distances competes with diffusion rates. Therefore, it is possible to associate with each effective encounter distance $R_{\text {eff }}$ a rate constant $k_{\text {et }}\left(R_{\text {eff }}\right)$ (sink term, $\left[A \exp \left(-2 R_{\text {eff }} / L\right)\right]$ ).

Rearrangement of eq 20 gives

$$
\exp \left(-2 R_{\mathrm{eff}} / L\right)=4 D /\left(\mathrm{A} L^{2} e^{2 E}\right)
$$

Therefore, at very high viscosities, the rate constant $k_{\mathrm{ct}}\left(R_{\mathrm{eff}}\right)$ can be evaluated by

$$
k_{\mathrm{ct}}\left(R_{\mathrm{eff}}\right)=4 D /\left(L^{2} e^{2 E}\right)
$$

Using eq 16 and 26 (for $D<4.0 \times 10^{-6} \mathrm{~cm}^{2} \mathrm{~s}^{-1}$ ), we obtained values of $k_{\text {ct }}$ which differ by a constant factor $f$ (Table IV), implying at these distances an equivalence of both models and the dependence of $k_{\mathrm{ct}}$ on $R_{\mathrm{eff}}, D$, and $\Delta G^{*}$. Therefore, the reactional distances determined in this work have the meaning of effective distances $R_{\text {eff }}$ which are related to an average value of $k_{\mathrm{ct}}(R)$ for a distribution function of each reactant pair whose separation distance is $R_{\mathrm{c}}<R<\infty$.

The treatment used involves several approximations such as the use of an average $L$, which in fact measures the overlap between 
the donor and acceptor at a given separation distance, and this should vary in each case. On the other hand, $k_{0}$ should vary with polarity, but yet the value obtained in more polar media such as micelles is of the same order of magnitude as the value of $k_{0}$ obtained in nonpolar mixtures of paraffin/heptane.

The distances obtained in nonionic micelles show some deviations from the ones calculated by eq 23, in particular for the system Bz-1-Pn/DMA. Since $R^{\prime}$ varies considerably with $D$, it is possible that at larger distances small variations in diffusion coefficients are more strongly felt. Also, the diffusion coefficient used, $D=$ $1.5 \times 10^{-6} \mathrm{~cm}^{2} \mathrm{~s}^{-1}$, was obtained from a homogeneous treatment $\mathrm{t}^{25}$ which when applied to micellar media may induce some errors.

The concurrent observation of an exciplex formation and estimation of effective reactional distances larger than collisional ones in nonpolar, viscous solvents may involve some precursor state in which the interaction, although very weak, is still sufficient to quench the fluorescence from ${ }^{1} \mathrm{~A}^{*}$ by electron transfer followed by slow relaxation to an exciplex with a minimum equilibrium separation of $\simeq 3 \times 10^{-8} \mathrm{~cm}$. Since we are dealing with exothermic systems, it is conceivable that the orbital integral overlap obtained for distances as large as $R_{\text {eff }}=10^{-7} \mathrm{~cm}$ (edge-to-edge difference of $3 \times 10^{-8} \mathrm{~cm}$ ), although very small, is sufficient to enable an electronic interaction in the pair $\left({ }^{1} A^{*} \ldots D\right)$. The charge transfer may then take place via excited vibrational states invoked by Jortner ${ }^{45}$ and Efrima and Bixon. ${ }^{46}$

The available data do not allow any distinction between a tunneling mechanism ${ }^{47}$ or an activated process with electronic mixing via virtual states which will contribute to the electron exchange before any encounter takes place.

Conclusion. The work presented here emphasizes the importance of redox potentials, polarity, and viscosities in processes which are in the diffusion-controlled limit. A distance dependence on the charge-transfer rate constant ${ }^{48}$ is demonstrated in viscous systems where the transfer at longer distances may dominate with a concomitant decrease in the dissociation rate constant of the encounter complex.

An equation for $k_{\mathrm{ct}}$ derived within the framework of a semiclassical theory $\left(K_{\mathrm{SC}}=K_{\mathrm{EL}}\langle\kappa\rangle \Gamma_{\mathrm{N}}\right){ }^{38}$ if one assumes a nonadiabatic regime without nuclear tunneling, is presented. The use of this equation allows an extension of Marcus formalism for rather exothermic systems where a small orbital overlap between the orbitals of the donor and acceptor is sufficient for reaction to occur

(45) J. Ulstrup and J. Jortner, J. Chem. Phys., 63, 4358 (1975).

(46) S. Efrima and M. Bixon, Chem. Phys., 13, 447 (1976).

(47) K. I. Zamaraev and R. F. Zainetdnov, Russ. Chem. Rev. (Engl. Transl.), 47, 518 (1978).

(48) R. A. Marcus, Faraday Discuss Chem. Soc., No. 74, 7 (1982). at effective "encounter" distances larger than true collisional ones. Furthermore, this work stresses the possibility of discussing the influence of parameters such as $k_{0}$ and $L$ in the magnitude of $k_{\mathrm{ct}}$, using simple equations such as (16) and (23). Namely, the parameter $L$ depends on the ionization potential and $k_{0}$ depends on the orbital overlap of the reactants and very likely also varies with $R$.

Consequently, the combination of these two factors may also be responsible for higher values of $k_{\mathrm{ct}}$ as those observed by Balzani ${ }^{40}$ with aromatic amines (lower ionization potential and $\pi$ aromatic system) as compared with aliphatic amines.

The experimental results obtained and the formalism presented in this paper seem to support the possibility of a unified treatment for charge-transfer processes which may or may not be diffusion controlled.

While there are several other models and treatments reported, ${ }^{49-52}$ we believe that expressions derived here are simple to use, requiring only experimental parameters which can be readily available for condensed media, and in addition they demonstrate the relative importance of various molecular and solvent parameters.

Acknowledgment. This work was supported by Instituto Nacional de Investigação Cientifica (I.N.I.C.) and in part by Junta Nacional de Investigação Cientifica e Tecnolōgica (J.N.I.C.T.).

\section{Appendix}

The term $Y\left(R^{\prime} D\right)$ is given by

$$
Y(x)=1-x \pi^{1 / 2} \exp x^{2} \operatorname{erfc} x
$$

with

$$
\begin{gathered}
x=b / a^{1 / 2} \quad \operatorname{erfc} x=2 / \pi^{1 / 2} \int_{x}^{\infty} e^{-\xi} \mathrm{d} \xi \\
a=1 / \tau_{0}+4 \pi N^{\prime} D R^{\prime}[\mathrm{Q}] \\
b=4 R^{\prime 2}(\pi D)^{-1 / 2} N^{\prime}[\mathrm{Q}]
\end{gathered}
$$

If $x$ is known, $Y(x)$ can be determined from curves of $Y(x)$ as a function of $x$.

Registry No. Bz-2-Np, 53531-11-6; Bz-9-Ant, 67777-65-5; Bz-1-Pn, 67777-66-6; TEQ, 121-44-8; DMA, 121-69-7; DABCO, 280-57-9; TMPD, 100-22-1.

(49) V. Balzani, F. Scandola, G. Orlandi, N. Sabbatini, and M. T. Indelli, J. Am. Chem. Soc., 103, 3370 (1981).

(50) R. K. Huddleston and J. R. Miller, J. Phys. Chem., 86, 200 (1982).

(51) J. Keiser, J. Phys. Chem., 86, 5052 (1982).

(52) S. Strauch, G. McLendon, M. McGuire, and T. Guarr, J. Phys. Chem., 87, 3579 (1983). 